\title{
A comparative study between sterile freeze-dried and sterile pre-hydrated acellular dermal matrix in tissue expander/implant breast reconstruction
}

\author{
Jeong Hyun Cheon, Eul Sik Yoon, Jin Woo Kim, Seung Ha Park, Byung Il Lee \\ Department of Plastic and Reconstructive Surgery, Korea University College Medicine, Seoul, Korea
}

Background In implant-based breast reconstruction, acellular dermal matrix (ADM) is essential for supporting the inferolateral pole. Recent studies have compared non-sterilized freeze-dried ADM and sterilized pre-hydrated ADM, but have not assessed whether differences were attributable to factors related to sterile processing or packaging. This study was conducted to compare the clinical outcomes of breast reconstruction using two types of sterile-processed ADMs. Methods Through a retrospective chart review, we analyzed 77 consecutive patients (85 breasts) who underwent tissue expander/implant breast reconstruction with either freezedried ADM (35 breasts) or pre-hydrated ADM (50 breasts) from March 2016 to February 2018. Demographic variables, postoperative outcomes, and operative parameters were compared between freeze-dried and pre-hydrated ADM. Biopsy specimens were obtained for histologic analysis.

Results We obtained results after adjusting for variables found to be significant in univariate analyses. The total complication rate for freeze-dried and pre-hydrated ADMs was $25.7 \%$ and $22.0 \%$, respectively. Skin necrosis was significantly more frequent in the freeze-dried group than in the pre-hydrated group ( $8.6 \%$ vs. $4.0 \%, \mathrm{P}=0.038$ ). All other complications and operative parameters showed no significant differences. In the histologic analysis, collagen density, inflammation, and vascularity were higher in the pre-hydrated $A D M$ group $(P=0.042, P=$ $0.006, P=0.005$, respectively).

Conclusions There are limited data comparing the outcomes of tissue expander/implant breast reconstruction using two types of sterile-processed ADMs. In this study, we found that using pre-hydrated ADM resulted in less skin necrosis and better integration into host tissue. Pre-hydrated ADM may therefore be preferable to freeze-dried ADM in terms of convenience and safety.

Keywords Breast implants / Acellular dermis / Mammaplasty / Sterilization

\author{
Correspondence: Eul Sik Yoon \\ Department of Plastic and \\ Reconstructive Surgery, Korea \\ University College Medicine, \\ 73 Inchon-ro, Seongbuk-gu, Seoul \\ 02841 , Korea \\ Tel: $+82-2-920-5368$ \\ Fax: +82-2-922-7437 \\ E-mail: yesanam2@korea.ac.kr
}

This article was presented at the PRS Korea 2017 on November 10-12, 2017, in Seoul, Korea.

\section{INTRODUCTION}

Two-stage implant-based breast reconstruction has emerged as a major option for patients who undergo mastectomy [1]. More than 50,000 tissue expander/implant-based breast reconstructions are performed annually in the United States, accounting 
for almost $60 \%$ of all postmastectomy breast reconstructions [2].

The use of acellular dermal matrix (ADM) in implant-based breast reconstruction is essential for obtaining a satisfactory contour by supporting the inferolateral pole, thereby enhancing early volume expansion. Various types of freeze-dried ADMs have been introduced, each with distinct characteristics (Table 1) [3].

Recently, pre-hydrated ready-to-use (RTU) ADM products have been introduced, as rehydrating the freeze-dried ADM before application was thought to be time-consuming for both patients and surgeons (Table 1, Fig. 1). Previous studies comparing pre-hydrated $\mathrm{ADM}$ and freeze-dried $\mathrm{ADM}$ showed that prehydrated $\mathrm{ADM}$ was more convenient to use than freeze-dried $\mathrm{ADM}$, without inferior outcomes [4-7]. In a more recent report, the use of freeze-dried ADM was associated with a significantly

\section{Fig. 1. Pre-hydrated acellular dermal matrix product}

MegaDerm HD (L\&C BIO, Seongnam, Korea) is initially stored in sterile normal saline, and can be used immediately without any additional hydration process.

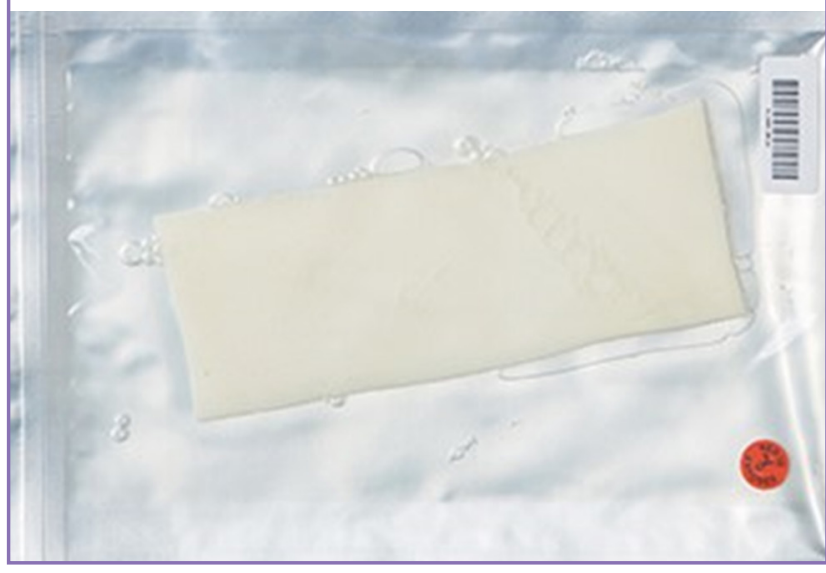

higher reconstructive failure rate than occurred when pre-hydrated $\mathrm{ADM}$ was used [8]. However, since all recent studies compared non-sterilized freeze-dried ADM with sterilized prehydrated $\mathrm{ADM}$, it was difficult to determine whether differences in outcomes resulted from factors related to sterile processing or packaging.

As freeze-dried and pre-hydrated ADMs are both produced using a sterile process by MegaDerm ( $\&$ C BIO), we were able to compare whether the freeze-drying process itself influenced outcomes, without interference from the sterilization process. The goal of this study was to compare the outcomes of breast reconstruction using either sterile freeze-dried $\mathrm{ADM}$ or sterile pre-hydrated $\mathrm{ADM}$, and to investigate whether there were any differences in the structure of the grafted matrix.

\section{METHODS}

\section{Patients}

Through a retrospective review, the records of all patients who underwent immediate tissue expander/implant breast reconstruction between March 2016 and February 2018 were analyzed. A senior surgeon (ESY) performed two-stage tissue expander/implant breast reconstruction procedures using two types (freeze-dried and pre-hydrated) of ADMs (MegaDerm; L\&C BIO, Seongnam, Korea) at a single institution. Freezedried ADM was used from March 2016 to December 2016, while pre-hydrated ADM was used from January 2017 to February 2018. Informed consent was obtained from patients, and this study was approved by the Institutional Review Board of Korea University Medical Center (IRB No. 2018AN0250). The exclusion criteria of the study were as follows: (1) patients undergoing a procedure using nonhuman $\mathrm{ADM}$, total muscle coverage of the tissue expander, direct-to-implant reconstruction,

\section{Table 1. Types of alloplastic adjuncts used in breast reconstruction}

\begin{tabular}{|lllll|}
\hline Product & \multicolumn{1}{c}{ Manufacturer } & Origin & Method of preservation & Sterility \\
\hline AlloDerm & Lifecell Corp. & Human dermis & Cryopreserved & Aseptic \\
AlloDerm RTU & Lifecell Corp. & Human dermis & Pre-hydrated & Sterilized \\
NeoForm & Mentor & Human dermis & Cryopreserved & Aseptic \\
Flex HD & Musculoskeletal Transplant Foundation and Ethicon & Human dermis & Pre-hydrated & Aseptic \\
AlloMax & Bard & Human dermis & Freeze-drying & Sterilized \\
DermaMatrix & Synthes & Human dermis & Freeze-drying & Aseptic \\
DermACELL & NOVADAQ & Human dermis & Pre-hydrated & Aseptic \\
Glyaderm & Euro Skin Bank & Human dermis & Cryopreserved & Aseptic \\
CollaMend & Bard & Porcine dermis & Cryopreserved & Aseptic \\
Permacol & Tissue Science Laboratories Porcine dermis & Porcine dermis & Cryopreserved & Sterilized \\
Strattice & Lifecell Corp. & Porcine dermis & Pre-hydrated & Sterilized \\
SurgiMend & TEl Biosciences & Bovine dermis & Pre-hydrated & \\
\hline RTU, ready-to-use. & & & & \\
\hline
\end{tabular}


or delayed reconstructions; (2) patients who had received radiation therapy before surgery and showed suspected clinical signs of radiation injury (i.e., pigmentation, induration, telangiectasia, or atrophy); and (3) severely obese patients, as defined by a body mass index $(\mathrm{BMI}) \geq 35 \mathrm{~kg} / \mathrm{m}^{2}$ or the presence of obesityrelated health conditions. BMI is known to have a major influence on the occurrence of complications, such as seroma and wound-related complications. The following patient-related variables were retrospectively obtained from their records: (1) demographic information (age and BMI), (2) clinical characteristics (smoking status, hypertension, diabetes, and obesity), and (3) neoadjuvant and/or adjuvant therapy use (preoperative and postoperative chemotherapy and/or radiation therapy). All outcome data were reviewed after the first-stage operation during expansion, and cases of infection, seroma, hematoma, skin necrosis, dehiscence or exposure, and explantation were identified. Seroma was considered to be present when surgery or ultrasound device-guided aspiration was necessary due to persistent drainage or the presence of erythema. Skin necrosis was defined as partial- or full-thickness skin necrosis requiring surgical debridement and repair. The total drainage amount, maintenance period of the drain, length of hospital stay, and operation time were recorded and analyzed to compare the inconvenience experienced by patients. All data were compared between the prehydrated and freeze-dried ADM groups.

\section{Surgical technique}

After a mastectomy operation by a general surgeon through an inframammary incision, a subpectoral dissection was performed and the inferior border of the pectoralis major muscle was released for an $\mathrm{ADM}$ sling. The newly created pocket was irrigated with betadine solution and normal saline. The size of the ADM was determined based on the dimensions of the defect, the size of the expander, and the base width. Most ADMs were $6 \times 16$ $\mathrm{cm}$. To prepare the freeze-dried ADM, the matrix was thawed and rehydrated in saline for at least 20 minutes. A tissue expander (Mentor Corp., Santa Barbara, CA, USA) was used in all patients. Its size ranged from 350 to $500 \mathrm{~mL}$ for each patient. Each $\mathrm{ADM}$ graft was circumferentially sutured to the inframammary fascia and major pectoralis muscle with a Vicryl 2-0 suture (Ethicon Inc., Somerville, NJ, USA) (Fig. 2). The skin was closed using the subcuticular method with Stratafix 4-0 sutures (Ethicon Inc.). Two closed-suction drains were placed into the peri$\mathrm{ADM}$ plane, one in the suprapectoral pocket and the other in the subpectoral pocket. Postoperatively, the drains were maintained in place for approximately 1 to 2 weeks depending on the volume of drainage. Then, the drains were removed when the volume of drainage was less than $30 \mathrm{~mL}$ for 2 consecutive days.

\section{Fig. 2. Intraoperative photo of acellular dermal matrix}

The acellular dermal matrix graft was circumferentially sutured to the inframammary fascia and major pectoralis muscle with a Vicryl 2-0 suture (Ethicon Inc., Somerville, NJ, USA).

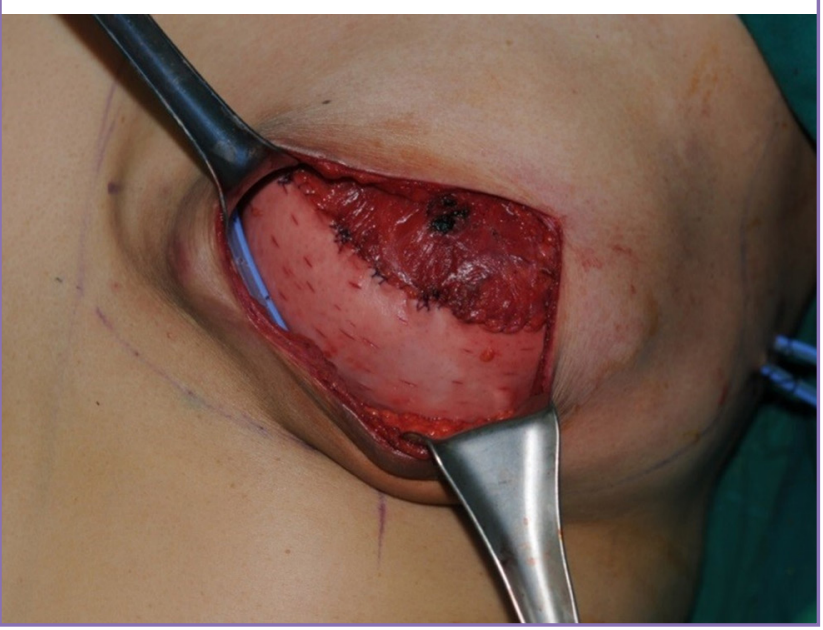

Postoperative intravenous antibiotics were used for 7 days during the same period in both groups. Postoperatively, expansion of the pocket began after complete wound healing. The volume and frequency of expansion were determined according to patients' comfort and tissue tolerance. Expansion did not exceed a volume $20 \%$ greater than the recommended volume of the expander. All breasts were reconstructed by placing a tissue expander that was exchanged 3 to 6 months later for a permanent implant.

\section{Histologic analysis}

During the second-stage operation, we approached the breast pocket via the inframammary line corresponding to the site of the previous incision. As the incorporated ADM was usually placed in the anterior flap of breast pocket, full-thickness tissue biopsy specimens measuring $1 \times 1 \mathrm{~cm}$ were obtained from the anterior flap. Biopsy samples were taken from the patients and sent to the pathology department in formalin for sectioning and staining. Hematoxylin and eosin staining was undertaken to assess general structure and cellularity. Masson trichrome staining was performed to assess the collagen fibers, and Verhoeff-Van Gieson staining was used to assess the elastin fibers. Additionally, immunohistochemical staining for the endothelial cell marker CD31 was performed to assess vascularity and neovascularization. Five biopsy results from each group were analyzed by routine light microscopy and reviewed by a pathologist (Bokyung Ahn: Department of Pathologic Korea University College Medicine, Korea). A semiquantitative scoring system (none, 0; mild, 1; moderate, 2 ; and severe, 3 ) was used to evaluate the biopsy results, including the presence of fibroblasts, chronic inflamma- 
tion, collagen and elastin fibers, and vessel proliferation. Scores were compared using the Mann-Whitney U test. Statistical significance was defined as $\mathrm{P}$-values $<0.05$.

\section{Statistical analysis}

Patients were divided into two groups based on the type of $\mathrm{ADM}$ used (freeze-dried, 30 patients, 35 breasts; pre-hydrated, 47 patients, 50 breasts). Data were extracted from the patients' medical records. Normally distributed data were expressed as mean \pm standard deviation, and non-normally distributed data were expressed as median (first quartile to third quartile). The patients' demographic variables were analyzed using the chisquare test or the Fisher exact test for categorical variables and the independent-samples t-test for numeric variables. As the postoperative outcome data were normally distributed, between-group comparisons were performed using the unpaired t-test. To adjust for variables found to be significant in the demographic analysis, the correlations between postoperative outcomes and smoking status and tissue expander size were assessed using logistic regression analysis. Because the data on operative parameters were non-normally distributed, betweengroup comparisons were performed using the Mann-Whitney $\mathrm{U}$ test. P-values $<0.05$ were considered to indicate statistical significance. SPSS version 20 (IBM Corp., Armonk, NY, USA) was used for all statistical tests.

\section{RESULTS}

A total of 77 patients ( 85 breasts) underwent mastectomy with two-stage implant-based breast reconstruction using $\mathrm{ADM}$. All reconstructions were immediate, concomitant implant-based breast reconstructions. Eight patients underwent bilateral surgery. Reconstruction using traditional freeze-dried ADM was performed for $41.18 \%$ of the breasts $(n=35)$, while $58.82 \%(n=$ 50) were reconstructed using pre-hydrated ADM (Table 2). The patients' average age was $48.6 \pm 9.07$ years. Their mean BMI was $23.1 \pm 3.29 \mathrm{~kg} / \mathrm{m}^{2}$. Eight patients were current smokers, only in the pre-hydrated group. The median follow-up time for patients was 4.7 months (range, 3.2-18 months). In our series, 49 patients received chemotherapy, including 40 patients who received chemotherapy after the breast was reconstructed. Radiotherapy was administered to 12 patients, including 10 patients to whom it was administered after breast reconstruction. During the operation, 60 patients underwent a sentinel lymph node biopsy procedure. Twenty of those patients required axillary node clearance at the time of the reconstruction. When the breasts reconstructed with traditional freeze-dried $\mathrm{ADM}$ were compared to those reconstructed with pre-hydrated $\mathrm{ADM}$, the patients
Table 2. Demographic and clinical characteristics of the breast reconstruction patients

\begin{tabular}{|c|c|c|c|}
\hline Characteristic & Freeze-dried & Pre-hydrated & P-value \\
\hline No. of patients & 30 & 47 & - \\
\hline No. of breasts & 35 & 50 & - \\
\hline Age (yr) & $47.7 \pm 3.2$ & $49.2 \pm 2.6$ & 0.455 \\
\hline $\mathrm{BMI}\left(\mathrm{kg} / \mathrm{m}^{2}\right)$ & $23.1 \pm 3.9$ & $22.6 \pm 2.7$ & 0.105 \\
\hline$<30$ & $34(97.1)$ & $49(98)$ & \\
\hline$\geq 30$ & $1(2.9)$ & $1(2)$ & \\
\hline Smoking (current) & 0 & $8(16)$ & 0.019 \\
\hline Diabetes & $2(5.7)$ & $4(8)$ & 0.243 \\
\hline Tissue expander size (mL) & & & $<0.001$ \\
\hline 350 & $7(20.0)$ & $34(68)$ & \\
\hline 400 & $23(65.7)$ & $5(10)$ & \\
\hline 450 & $1(2.9)$ & $9(18)$ & \\
\hline 500 & $4(11.4)$ & $2(4)$ & \\
\hline Mastectomy & & & 0.574 \\
\hline Unilateral & $25(71.4)$ & $42(84)$ & \\
\hline Bilateral & $5(28.6)$ & $4(16)$ & \\
\hline Axillary lymph node dissection & $11(31.4)$ & $9(18)$ & 0.292 \\
\hline Sentinel lymph node biopsy & $23(65.7)$ & $37(74)$ & 0.292 \\
\hline Chemotherapy & & & 0.827 \\
\hline Pre-reconstruction & 0 & $9(18)$ & \\
\hline Post-reconstruction & $17(48.5)$ & $23(46)$ & \\
\hline Radiotherapy & & & 0.540 \\
\hline Pre-reconstruction & $1(2.9)$ & $1(2)$ & \\
\hline Post-reconstruction & $6(17.1)$ & $4(8)$ & \\
\hline Hormone therapy & $21(60.0)$ & $32(64)$ & 0.271 \\
\hline \multicolumn{4}{|c|}{$\begin{array}{l}\text { Values are presented as mean } \pm S D \text { or number (\%). Data comparison was } \\
\text { performed using the chi-square test or the Fisher exact test for categorical } \\
\text { variables and the independent-samples t-test for numeric variables. }\end{array}$} \\
\hline
\end{tabular}

were similar in terms of age, BMI status, medical comorbidities, sentinel lymph node biopsy, axillary lymph node dissection, indication for surgery, need for adjuvant/neoadjuvant antineoplastic treatment, hormone therapy, and radiotherapy. However, the percentage of smokers among the patients whose breasts were reconstructed with pre-hydrated $\mathrm{ADM}$ was higher $(16 \%$, $\mathrm{n}=8$ ) than among those in whom freeze-dried ADM was used $(0 \%, \mathrm{n}=0)$. In addition, the size of the tissue expanders differed between the two groups (Table 2).

All surgical procedures were successful, without any problems. The total complication rates for the freeze-dried and pre-hydrated ADM groups were $25.71 \%$ and $22.0 \%$, respectively. The postoperative seroma rate was $8 \%$ in the pre-hydrated $\mathrm{ADM}$ group and $11.42 \%$ in the freeze-dried $\mathrm{ADM}$ group $(\mathrm{P}=0.596)$. The overall infection rate was $12 \%$ in the pre-hydrated $\mathrm{ADM}$ group and $8.57 \%$ in the freeze-dried ADM group $(\mathrm{P}=0.615)$. However, the rate of infection was not significantly different between the two groups. Postoperative infections occurred in three patients $(8.57 \%)$ in the freeze-dried ADM group, including two cases $(5.71 \%)$ of infection requiring conservative treat- 
Table 3. Postoperative outcomes of study participants

\begin{tabular}{|c|c|c|c|c|c|}
\hline & Freeze-dried $(n=35)$ & Pre-hydrated $(n=50)$ & Odds ratio & P-value & Adjusted P-value ${ }^{\text {a) }}$ \\
\hline Complications (total) $)^{b}$ & $9(25.71)$ & $11(22)$ & 0.815 & 0.691 & 0.847 \\
\hline Seroma & $4(11.42)$ & $4(8)$ & 0.674 & 0.596 & 0.596 \\
\hline Tissue expander migration & $1(2.86)$ & $1(2)$ & 0.694 & 0.799 & 0.799 \\
\hline Hematoma & $1(2.86)$ & $3(6)$ & 2.170 & 0.510 & 0.510 \\
\hline Infection & $3(8.57)$ & $6(12)$ & 1.455 & 0.615 & 0.392 \\
\hline Requiring readmission & $2(5.71)$ & $4(8)$ & 1.435 & 0.687 & 0.687 \\
\hline Requiring surgery & $1(2.86)$ & $2(4)$ & 1.417 & 0.780 & 0.896 \\
\hline Exposure/dehiscence & $2(5.71)$ & $1(2)$ & 0.337 & 0.382 & 0.382 \\
\hline Skin margin necrosis & $7(20.00)$ & $2(4)$ & 0.167 & $0.032^{c)}$ & $0.032^{c)}$ \\
\hline Explantation & $1(2.86)$ & $1(2)$ & 0.694 & 0.799 & 0.799 \\
\hline
\end{tabular}

Table 4. Operative parameters of study participants

\begin{tabular}{|lccc|}
\hline & Freeze-dried $(\boldsymbol{n}=\mathbf{3 5})$ & Pre-hydrated $(\mathrm{n}=\mathbf{5 0})$ & P-value \\
\hline Total amount of drainage $(\mathrm{mL})$ & $862.2(759.0-1,068.6)$ & $984(731.0-1,252.3)$ & 0.583 \\
Period of drainage (day) & $13(12-14)$ & $14(12-15)$ & 0.456 \\
Hospital day (day) & $15(13.5-16.0)$ & $14.5(10.3-16.0)$ & 0.120 \\
Operating time (min) & $125(90-147)$ & $105(106-145)$ & 0.668 \\
\hline Values are presented as median (first quartile to third quartile). Data comparison was performed using the Mann-Whitney U test. & \\
\hline
\end{tabular}

ment with intravenous antibiotics and one case $(2.86 \%)$ of infection requiring surgery. In the pre-hydrated ADM group, postoperative infections occurred in six cases (12\%), including four cases $(8 \%)$ of infection requiring conservative treatment with intravenous antibiotics and two cases (4\%) of infection requiring surgery. There were more postoperative infections requiring surgery in the pre-hydrated ADM group, but the difference between the two groups was not statistically significant $(\mathrm{P}=0.896)$. The overall incidence of skin necrosis was $4 \%$ ( 2 cases) in the pre-hydrated ADM group and 20\% (7 cases) in the freeze-dried ADM group. This discrepancy between the two groups was statistically significant $(\mathrm{P}=0.032)$. Despite skin necrosis, five patients had successful results, with good to excellent cosmetic outcomes and well-vascularized and well-incorporated ADMs. The rates of other complications, including hematoma, tissue expander migration, exposure/wound dehiscence, and explantation, were equivalent between the two groups (Table 3).

Smoking status and tissue expander size were found to be significant variables in the demographic analysis (Table 2). However, smoking status and tissue expander size did not affect the postoperative outcomes, and our results remained significant after adjusting for these factors.

The median amount of drainage in patients with freeze-dried $\mathrm{ADM}$ was $862.2 \mathrm{~mL}$ (first quartile to third quartile, 759.0-
$1,068.0 \mathrm{~mL}$ ) and the median period of drainage was 13 days (first quartile to third quartile, 12-14 days). For patients with pre-hydrated $\mathrm{ADM}$, the median amount of seroma drainage was $984 \mathrm{~mL}$ (first quartile to third quartile, $731.0-1,252.3 \mathrm{~mL}$ ) and the median period of drainage was 14 days (first quartile to third quartile, 12-15 days) (Table 4). Patients whose breasts were reconstructed with $\mathrm{ADM}$ showed similar total drainage amounts and drain maintenance period regardless of type (pre-hydrated $\mathrm{ADM}$ or freeze-dried ADM). The length of hospital stay and operation time were not significantly different between the two groups.

At the time of biopsy, on gross visual inspection, all ADMs were fully taken within the host tissue. Both types were well adhered. The samples obtained $5 \pm 1.4$ months after the first-stage operation showed no significant differences between the two groups. The biopsy sample results from the second-stage operation showed that these two ADM types had similar forms of capsules and synovia-like metaplasia (Fig. 3). In the analysis using a semiquantitative scoring system, higher levels of chronic inflammation, neovascularization, and dense collagen were observed in the capsules from the pre-hydrated ADM group (Table 5). The median scores for chronic inflammation, collagen, and vessel proliferation in the patients who received freeze-dried $\mathrm{ADM}$ were 1 (range, $0-1$ ), 1 (range, 1-2), and 0 (range, $0-1$ ), 
Fig. 3. Histology of freeze-dried and pre-hydrated ADM

Both specimens were taken 5 months after placement during second-stage tissue expander-to-implant exchange. Compared with (A) the freeze-dried group $(H \& E, \times 40)$, the $(B)$ pre-hydrated group showed more chronic inflammatory cells (blue). Compared with (C) the freeze-dried group (Masson trichrome staining, $\times 40)$, (D) the collagen fibers (blue) in the pre-hydrated group were denser. In both groups-(E) the freeze-dried group (VerhoeffVan Gieson staining, $\times 40)$ and (F) the pre-hydrated group-elastin fibers (black color: yellow arrows) were observed. Compared with $(\mathrm{G})$ the freeze-dried group (CD31 immunohistochemical staining, $\times 40),(H)$ the vessels were more visible in the pre-hydrated group. The lumens of blood vessels can be appreciated (asterisks). ADM, acellular dermal matrix.
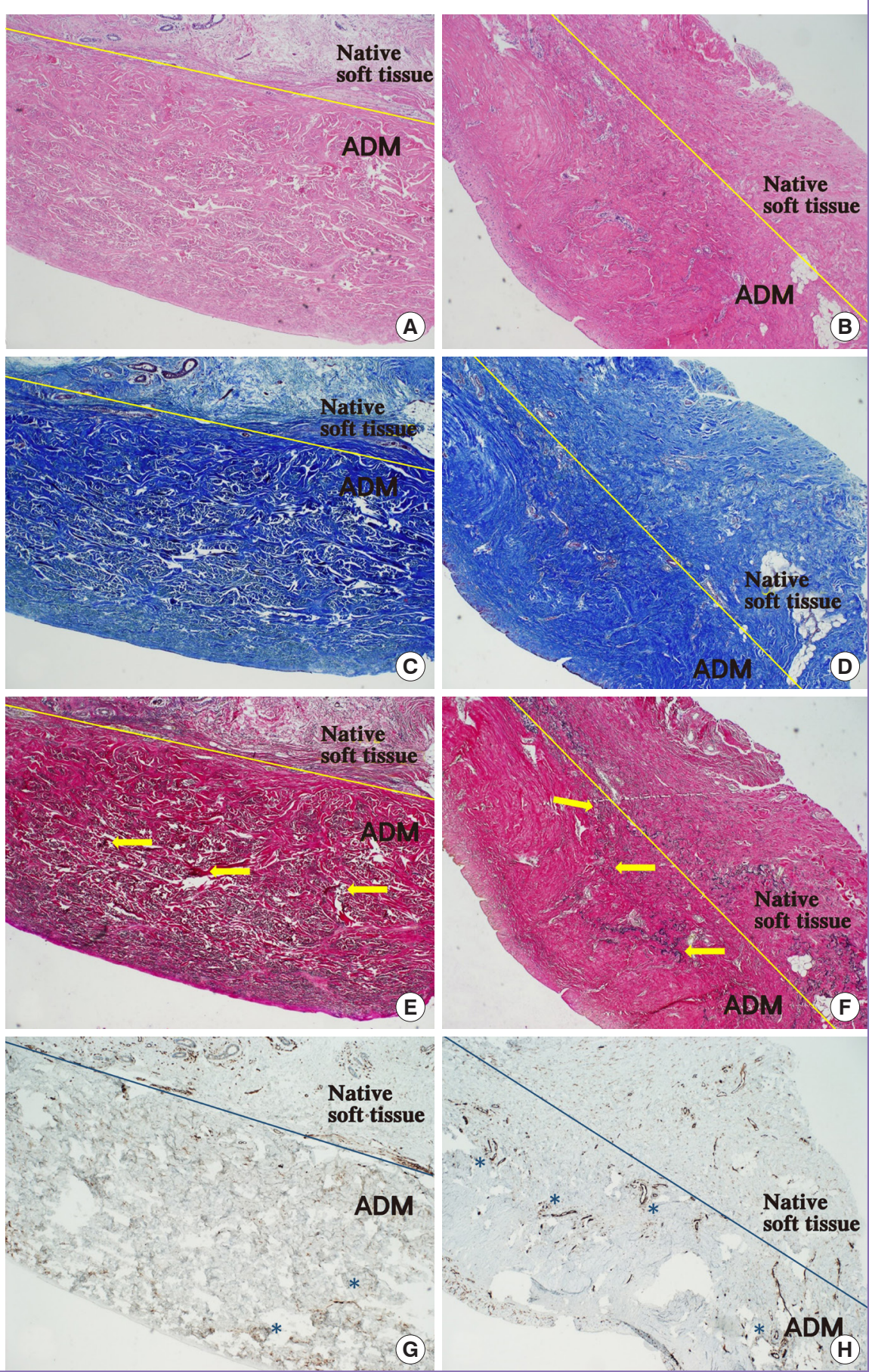

respectively. For patients who received pre-hydrated $\mathrm{ADM}$, the median scores for chronic inflammation, collagen, and vessel proliferation were 2 (range, 2-3), 2 (range, 2-3), and 3 (range, $2-3)$, respectively.

\section{DISCUSSION}

The use of $\mathrm{ADM}$ as an adjuvant to implant-based reconstruc- tion may overcome the limitations of traditional subpectoral or dual-plane prosthetic breast reconstruction and improve the overall aesthetic outcomes with an acceptable complication rate [9]. It has also been found to reduce the risk of capsular contracture and implant malposition to a significant extent, compared to the subpectoral technique [10]. Using ADMs can provide additional reinforcement. Thus, less time is required to complete the mastectomy pocket and final reconstruction [10]. 
Table 5. Statistical comparison of the results of histologic evaluations scored on a point-based system

\begin{tabular}{|c|c|c|c|c|c|c|c|c|c|c|c|}
\hline & \multicolumn{5}{|c|}{ Score of freeze-dried group $(n=5)$} & \multicolumn{5}{|c|}{ Score of pre-hydrated group $(n=5)$} & \multirow{2}{*}{ P-value } \\
\hline & 0 & 1 & 2 & 3 & Median & 0 & 1 & 2 & 3 & Median & \\
\hline Fibroblasts & 0 & 4 & 1 & 0 & 1 & 0 & 1 & 2 & 2 & 2 & 0.540 \\
\hline Chronic inflammation & 2 & 3 & 0 & 0 & 1 & 0 & 0 & 4 & 1 & 2 & $0.006^{a)}$ \\
\hline Collagen & 0 & 3 & 2 & 0 & 1 & 0 & 0 & 4 & 1 & 2 & $0.042^{\mathrm{a})}$ \\
\hline Elastin & 0 & 1 & 2 & 2 & 2 & 0 & 0 & 2 & 3 & 3 & 0.419 \\
\hline Vessel proliferation & 4 & 1 & 0 & 0 & 0 & 0 & 0 & 1 & 4 & 3 & $0.005^{a)}$ \\
\hline
\end{tabular}

These ADMs are derived from donated human cadaveric dermis via processing to remove cellular elements. The current $\mathrm{ADMs}$ available for breast reconstruction are classified as prehydrated or freeze-dried according to the processing method, or as sterile or aseptic according to the sterilization technique. Specific processing techniques are used for each type of $\mathrm{ADM}$, resulting in different characteristics and presentation of tissue forms in pre-existing products [11]. Emerging data appear to link ADM utilization with high rates of total complications, including infections, seroma, and reconstructive failure [9,12-16]. Given concerns over the possibility of elevated complication rates, risk stratification for individual types of $\mathrm{ADM}$ is rare.

The most commonly used ADMs are freeze-dried. They can be used after thorough hydration with normal sterile saline for 20 to 40 minutes. The biomechanical properties of collagenous grafts can change significantly when the rehydration time increases [17]. Such changes have also been confirmed structurally through physicochemical analyses [17]. Therefore, the rehydration time should be at least 20 minutes. Since the appropriate size of $\mathrm{ADM}$ is often determined during surgery, the rehydration process significantly affects the length of surgery. Especially in cases of abrupt use or size change, this process can become time-consuming and increase the patient's risk of exposure to infection in the operating room.

Pre-hydrated ADMs are utilized with increasing frequency for breast reconstruction because they can shorten the preparation time. The processing technique used for pre-hydrated ADMs results in a product that is fully hydrated throughout preparation and packaging; therefore, it can be used immediately, with no time required for rehydration. Pre-hydrated ADMs have been described in breast reconstruction studies in humans and animals $[7,11]$. However, in these studies, most comparisons were made between pre-hydrated ADM and aseptic freeze-dried $\mathrm{ADM}$, not sterile freeze-dried ADM, because most freeze-dried ADMs are aseptic.

Buseman et al. [5] performed a comparative study of aseptic freeze-dried $\mathrm{ADM}$ and sterile pre-hydrated $\mathrm{ADM}$ and found that sterile pre-hydrated $\mathrm{ADM}$ had a risk of infection equivalent to that of aseptic freeze-dried ADM. However, patients in the sterile pre-hydrated ADM group showed a higher incidence of seroma than patients in the aseptic freeze-dried ADM group. Khansa et al. [18] performed a retrospective review of cases where pre-hydrated $\mathrm{ADM}$ or cryopreserved $\mathrm{ADM}$ was used, and found no significant differences in complication outcomes between the two products. In addition, Yuen et al. [4] demonstrated a clinically higher postoperative complication rate in an obese population in immediate breast reconstruction with RTU AlloDerm than in procedures using freeze-dried AlloDerm. A meta-analysis by Macarios et al. [6] demonstrated that RTU and freeze-dried ADMs had similar infection, seroma, and explantation outcomes when they were used for prosthetic breast reconstruction. Weichman et al. [19] examined 90 breast reconstructions using cryopreserved AlloDerm and 105 breasts with RTU AlloDerm and found infection rates of $20 \%$ in the freezedried group and $8.5 \%$ in the RTU AlloDerm group.

The human ADMs used in this study were decellularized and terminally sterilized using an electron beam for freeze-dried $\mathrm{ADMs}$ and low-dose gamma irradiation for pre-hydrated $\mathrm{ADMs}$ [20]. Both products were prepared using a sterilization process. The sterile $\mathrm{ADM}$ was terminally sterilized to achieve a sterility assurance level of $10^{-3}$. To the best of our knowledge, no study has compared sterile, freeze-dried ADM with sterile, pre-hydrated ADM. Lee et al. [21] showed that sterile ADM was not associated with the infection rate by directly comparing aseptic freeze-dried products with sterile freeze-dried products. In contrast, Venturi et al. [22] showed that the sterilization process offered reliable matrix incorporation with a low complication rate. Since there is no consensus about the effects of the sterilization process, the direct comparison presented in this paper is meaningful.

Pre-hydrated ADM was associated with a lower rate of seroma occurrence in this study, although this difference did not reach statistical significance. Based on our results, we believe that recently introduced pre-hydrated ADM could minimize damage 
to 3-dimensional dermal structures because there is no freezing or drying process. For freeze-dried ADM, ice crystals inevitably form in the freezing process and the dermal matrix is destroyed. In the drying process, tensile strength is weakened due to the breakage of hydrogen bonds, leading ultimately to the loss of the collagen triple-helix structure [23]. We also believe that pre-hydrated ADM is less prone to destruction of these structures. Thus, the period of integration (usually approximately 2 weeks) may be shorter and seroma may be less common. In fact, when the pocket was inspected clinically, pre-hydrated $\mathrm{ADM}$ was often more difficult to remove (data not shown). The cause of seroma formation varies; however, dead space formation due to a mismatch that occurs prior to $\mathrm{ADM}$ integration is an important reason.

Buseman et al. [5] and Yuen et al. [4] showed that the use of sterile RTU ADM was associated with a statistically significant increase in seroma formation. This is in contrast to our study, which found no significant between-group differences in seroma occurrence. Interestingly, considering the differences between other publications and our study, these findings suggest that seroma may be related to the sterilization process. However, the etiology of this increased incidence remains unknown, and additional studies are needed to determine the mechanisms involved.

Our results showed statistically significant differences between the two groups in terms of skin necrosis, which was more common in the freeze-dried group. Other complications, such as hematoma, tissue expander migration, exposure/wound dehiscence, and explantation, were not statistically significantly different between the two groups. In our histologic analysis, higher levels of dense collagen, more red blood cells, and greater levels of chronic inflammation were observed in the pre-hydrated ADM group. Fibrovascular ingrowth into an implanted biomaterial is an indicator of incorporation and suggests better longterm retention without complications [24]. As such, the tissue biopsy results corresponded to our clinical observations. The greater angiogenesis and denser fibrocollagenous tissue observed in the biopsy results for pre-hydrated ADM might have contributed to flap stability. The revascularization of the mastectomy flap and the prolonged integration process may stimulate a host inflammatory response.

Antony et al. [12] reported that only age, BMI, and axillary dissection were found to be significant independent risk factors for the development of complications after ADM/tissue-expander breast reconstruction. These results are consistent with our findings. In our correlation analysis, smoking status did not affect postoperative outcomes. Another study conducted by Goodwin et al. [25] showed that patients who smoked were three times more likely to experience mastectomy flap necrosis than those who had never smoked. A major strength of this study is that it showed fewer cases of skin flap necrosis despite a higher prevalence of smoking in the pre-hydrated group. However, since this is an indirect comparison, whether the smoking variable actually reinforced our results is unknown.

Our study has several limitations, including its retrospective nature, which may have caused selection bias and nonrandomized case assignments. The small sample size is another limitation. Thus, further studies with larger cohorts are needed. In our study, although most conditions were similar between both groups, some factors might have influenced the outcomes. For example, our study did not address comparative outcomes according to the initial volume filling, although it has been reported that filling could reduce seroma by eliminating dead space [7]. In a previous study, the use of $\mathrm{ADM}$ enabled significant initial intraoperative tissue expander filling, representing $60 \%$ of the final tissue expander fill volume on average. The thickness of the skin flap after skin-sparing mastectomy or nipple-sparing mastectomy may be another factor because too thin of a skin flap can cause infection or skin necrosis. Two breast surgeons participated in our study. The operating time and thickness of the skin flap might have differed between the surgeons.

To minimize the influence of these biases on the outcomes of tissue expander breast reconstruction, similar well-designed prospective studies are needed to reach a consensus on the effects of ADM implantation. Future in vitro cell studies and studies of biomechanical characteristics should be performed to confirm our results and to help determine the underlying causes. Future studies examining potential differences in the economic impact between freeze-dried and pre-hydrated ADM use for breast reconstructions are also warranted.

There are limited data comparing the outcomes of tissue expander/implant breast reconstruction using two types of sterileprocessed ADMs. In this study, we found that pre-hydrated ADM showed less skin necrosis than freeze-dried ADM. Furthermore, in the histologic analysis, pre-hydrated ADM showed higher collagen density and more red blood cells in the specimens. These data suggest that pre-hydrated ADM might exhibit better integration into host tissue than freeze-dried ADM. Prehydrated ADM could therefore be preferable to freeze-dried $\mathrm{ADM}$ in terms of convenience and safety.

\section{NOTES}

\section{Conflict of interest}

No potential conflict of interest relevant to this article was reported. 


\section{Ethical approval}

The study was approved by the Institutional Review Board of Korea University Medical Center (IRB No. 2018AN0250) and performed in accordance with the principles of the Declaration of Helsinki. Written informed consent was obtained.

\section{Patient consent}

The patients provided written informed consent for the publication and the use of their images.

\section{Author contribution}

Study concept and design: Yoon ES. Data analysis and interpretation, drafting of the manuscript: Cheon JH. Critical revision of the manuscript for important intellectual content: Lee BI, Park SH. Technical, surgical support: Kim JW.

\section{ORCID}

Jeong Hyun Cheon https://orcid.org/0000-0003-3979-7240 Eul Sik Yoon https://orcid.org/0000-0001-5734-6625

Jin Woo Kim https://orcid.org/0000-0002-9012-7042

Seung Ha Park https://orcid.org/0000-0002-2667-2791

Byung Il Lee https:/ /orcid.org/0000-0001-8183-0388

\section{REFERENCES}

1. Albornoz CR, Bach PB, Mehrara BJ, et al. A paradigm shift in U.S. breast reconstruction: increasing implant rates. Plast Reconstr Surg 2013;131:15-23.

2. American Society of Plastic Surgeons. 2016 Plastic surgery procedural statistics [Internet]. Arlington Heights, IL: American Society of Plastic Surgeons; c2017 [cited 2019 Mar 31]. Available from https://www.plasticsurgery.org/ documents/News/Statistics/2016/plastic-surgery-statistics-full-report-2016.pdf.

3. Chao AH. A review of the use of acellular dermal matrices in postmastectomy immediate breast reconstruction. Plast Surg Nurs 2015;35:131-4.

4. Yuen JC, Yue CJ, Erickson SW, et al. Comparison between freeze-dried and ready-to-use AlloDerm in alloplastic breast reconstruction. Plast Reconstr Surg Glob Open 2014;2:e119.

5. Buseman J, Wong L, Kemper P, et al. Comparison of sterile versus nonsterile acellular dermal matrices for breast reconstruction. Ann Plast Surg 2013;70:497-9.

6. Macarios D, Griffin L, Chatterjee A, et al. A meta-analysis assessing postsurgical outcomes between aseptic and sterile AlloDerm regenerative tissue matrix. Plast Reconstr Surg Glob Open 2015;3:e409.

7. Rawlani V, Buck DW 2nd, Johnson SA, et al. Tissue expand- er breast reconstruction using prehydrated human acellular dermis. Ann Plast Surg 2011;66:593-7.

8. Hanson SE, Meaike JD, Selber JC, et al. Aseptic freeze-dried versus sterile wet-packaged human cadaveric acellular dermal matrix in immediate tissue expander breast reconstruction: a propensity score analysis. Plast Reconstr Surg 2018; 141:624e-632e.

9. Sbitany H, Sandeen SN, Amalfi AN, et al. Acellular dermisassisted prosthetic breast reconstruction versus complete submuscular coverage: a head-to-head comparison of outcomes. Plast Reconstr Surg 2009; 124:1735-40.

10. Lee KT, Mun GH. Updated evidence of acellular dermal matrix use for implant-based breast reconstruction: a metaanalysis. Ann Surg Oncol 2016;23:600-10.

11. Roth JS, Dexter DD, Lumpkins K, et al. Hydrated vs. freezedried human acellular dermal matrix for hernia repair: a comparison in a rabbit model. Hernia 2009;13:201-7.

12. Antony AK, McCarthy CM, Cordeiro PG, et al. Acellular human dermis implantation in 153 immediate two-stage tissue expander breast reconstructions: determining the incidence and significant predictors of complications. Plast Reconstr Surg 2010;125:1606-14.

13. Lanier ST, Wang ED, Chen JJ, et al. The effect of acellular dermal matrix use on complication rates in tissue expander/ implant breast reconstruction. Ann Plast Surg 2010;64:6748.

14. Chun YS, Verma K, Rosen H, et al. Implant-based breast reconstruction using acellular dermal matrix and the risk of postoperative complications. Plast Reconstr Surg 2010;125: 429-36.

15. Preminger BA, McCarthy CM, Hu QY, et al. The influence of AlloDerm on expander dynamics and complications in the setting of immediate tissue expander/implant reconstruction: a matched-cohort study. Ann Plast Surg 2008;60: 510-3.

16. Kim JY, Davila AA, Persing S, et al. A meta-analysis of human acellular dermis and submuscular tissue expander breast reconstruction. Plast Reconstr Surg 2012;129:28-41.

17. Bottino MC, Jose MV, Thomas V, et al. Freeze-dried acellular dermal matrix graft: effects of rehydration on physical, chemical, and mechanical properties. Dent Mater 2009;25: 1109-15.

18. Khansa I, Hendrick RG Jr, Shore A, et al. Breast reconstruction with tissue expanders: implementation of a standardized best-practices protocol to reduce infection rates. Plast Reconstr Surg 2014;134:11-8.

19. Weichman KE, Wilson SC, Saadeh PB, et al. Sterile "readyto-use" AlloDerm decreases postoperative infectious com- 
plications in patients undergoing immediate implant-based breast reconstruction with acellular dermal matrix. Plast Reconstr Surg 2013;132:725-36.

20. Moore MA, Samsell B, Wallis G, et al. Decellularization of human dermis using non-denaturing anionic detergent and endonuclease: a review. Cell Tissue Bank 2015;16:249-59.

21. Lee JH, Park Y, Choi KW, et al. The effect of sterile acellular dermal matrix use on complication rates in implant-based immediate breast reconstructions. Arch Plast Surg 2016;43: 523-8.

22. Venturi ML, Mesbahi AN, Boehmler JH 4th, et al. Evaluating sterile human acellular dermal matrix in immediate ex- pander-based breast reconstruction: a multicenter, prospective, cohort study. Plast Reconstr Surg 2013;131:9e-18e.

23. Bachmann L, Gomes AS, Zezell DM. Collagen absorption bands in heated and rehydrated dentine. Spectrochim Acta A Mol Biomol Spectrosc 2005;62:1045-9.

24. Eppley BL. Experimental assessment of the revascularization of acellular human dermis for soft-tissue augmentation. Plast Reconstr Surg 2001;107:757-62.

25. Goodwin SJ, McCarthy CM, Pusic AL, et al. Complications in smokers after postmastectomy tissue expander/implant breast reconstruction. Ann Plast Surg 2005;55:16-9. 